Pacific Journal of Mathematics

CONTINUITY OF MODULE AND HIGHER DERIVATIONS 


\title{
CONTINUITY OF MODULE AND HIGHER DERIVATIONS
}

\author{
Nicholas P. JeWELL
}

In this paper it is shown that derivations from $L^{1}[0,1]$ into Banach- $L^{1}[0,1]$-modules are automatically continuous. The result is obtained as a corollary of a theorem in which sufficient conditions on the closed ideals of a separable commutative Banach algebra $B$ are given so that every module derivation from $B$ into a Banach- $B$-bimodule is continuous. One of the conditions obtained is best possible. For the general case of a Banach algebra $A$ (not necessarily commutative or separable) sufficient conditions on the closed ideals are also given to force the continuity of module derivations and of certain higher derivations from any Banach algebra onto $A$.

1. Introduction. It is known that module derivations from a $C^{*}$-algebra $A$ into a Banach- $A$-bimodule are continuous [14]. We prove that module derivations from the radical Banach algebra $L^{1}[0,1]$ with convolution product [1] into a Banach- $L^{1}[0,1]$-module are continuous. This result is obtained as a corollary of Theorem 5 in which sufficient conditions on a separable commutative Banach algebra $B$ are given so that every module derivation from $B$ into a $B$ anach- $B$-bimodule is continuous. A well-known example shows that one of these conditions is best possible. Theorem 2 gives weaker sufficient conditions for the general case when $B$ need not be commutative or separable. In this situation W. G. Bade and P. C. Curtis, Jr. [2] have obtained slightly different sufficient conditions. However, using the example $L^{1}[0,1]$, we show that the results are in fact different. Also, in Theorem 7, we give sufficient conditions on a Banach algebra $B$ so that certain higher derivations from a Banach algebra onto $B$ are continuous. Our proofs are given for algebras over the complex field, but the results hold for real Banach algebras also. For the definition and elementary properties of Banach bimodules we refer to [4]. Throughout ideals are twosided. For $A$ a Banach algebra, $A^{k}$ is the ideal spanned by $k$-fold products of elements of $A$.

Recall that if $B$ is a Banach algebra and $M$ a Banach- $B$-bimodule then a linear map $D: B \rightarrow M$ is a module derivation if $D(a b)=$ $a \cdot D(b)+D(a) \cdot b$ for all $a, b$ in $B$ (where - denotes the module operation on $M$ ).

For $m$ in $\underset{\sim}{N}$, a higher derivation of rank $m$ (respectively infinite rank) 
from an algebra $A$ into an algebra $B$ is a sequence $\left\{F_{0}, \cdots, F_{m}\right\}$ (resp. $\left.\left\{F_{0}, F_{1} \cdots\right\}\right)$ of linear operators from $A$ into $B$ satisfying $F_{n}(a b)=$ $\sum_{l=0}^{n} F_{i}(a) F_{n-l}(b)$ for each $n=0,1, \cdots, m$ (resp. $\left.n=0,1,2, \cdots\right)$ and all $a, b$, in $A$.

A higher derivation of rank $m$ (resp. infinite rank) is said to be continuous if $F_{n}$ is continuous on $A$ for each $n=0,1, \cdots, m$ (resp. $n=0,1,2, \cdots)$. It is said to be onto if $F_{0}$ maps $A$ onto $B$.

The methods we use are extensions of those appearing in [8].

Throughout this note $\subset$ means strict inclusion. I am grateful to A. M. Sinclair for several stimulating conversations on the subject of automatic continuity.

2. Module derivations. In this section we establish sufficient conditions on the closed ideals of a Banach algebra $B$ so that every module derivation on $B$ is continuous. When $B$ is separable and commutative we can improve one of the conditions which then becomes best possible.

If $S$ is a linear operator from a Banach space $X$ into a Banach space $Y$, then the separating space, $\widetilde{\Xi}(S)$, of $S$ is defined by $\widetilde{S}(S)=\{y \in Y$ : there are $x_{n} \rightarrow 0$ in $x$ with $\left.S x_{n} \rightarrow y\right\}$. $\subseteq(S)$ gives us a measure of the discontinuity of the linear operator $S$ since the closed graph theorem shows that $S$ is continuous if and only if $\subseteq(S)=\{0\}$.

The following stability lemma for the separating space is the crucial tool we use for proving our main result.

Lemma 1. Let $X$ and $Y$ be Banach spaces and let $\left\{T_{n}\right\}$ and $\left\{R_{n}\right\}$ be sequences of continuous linear operators on $X$ and $Y$, respectively. If $S$ is a linear operator from $X$ into $Y$ such that $R_{n} S-S T_{n}$ is continuous for all $n$, then there is an integer $N$ such that $\left(R_{1} \cdots R_{n} \subseteq(S)\right)^{-}=\left(R_{1} \cdots R_{N} \subseteq(S)\right)^{-}$ for $n \geqq N$.

Proof. See [8, Lemma 1].

We note that if $X, Y,\left\{T_{n}\right\},\left\{R_{n}\right\}, S, N$ are as in Lemma 1 , and $\left\{U_{n}\right\}$ is a sequence of continuous linear operators on $Y$ such that $U_{n} R_{1} \cdots R_{n} \subseteq(S)=\{0\}$ for all $n$ then $U_{n} R_{1} \cdots R_{n-1} \subseteq(S)=\{0\}$ for all $n>N$. This is essentially remark 3.1 of [16].

Let $B$ be a Banach algebra, $M$ a Banach- $B$-bimodule and $D$ a module derivation from $B$ into $M$. We define $I_{L}=\{b \in B: b \cdot \subseteq(D)=$ 
$\{0\}\}$ and $I_{R}=\{b \in B: \subseteq(D) \cdot b=\{0\}\}$. It is easy to see that $I_{L}$ and $I_{R}$ are closed ideals of $B$ [2]. Proposition 2.4 of [2] shows that if $I_{L}$ has a bounded left (or right) approximate identity then $D$ is continuous on $I_{L}$. We call $I_{L}$ (and $I_{R}$ ) the left (and right) continuity ideal for $D$. If $B$ is commutative then $I_{L}=I_{R}$ and in this case we denote the ideal by $I$ and refer to it as the continuity ideal for $D$.

THEOREM 2. Let $B$ be a Banach algebra which satisfies the following conditions.

(1) If $K$ is a closed ideal of infinite codimension in $B$, then there exist sequences $\left\{b_{n}\right\},\left\{c_{n}\right\}$ in $B$ satisfying $c_{n} b_{1} \cdots b_{n-1} \notin K$ and $c_{n} b_{1} \cdots b_{n} \in K$ for all $n \geqq 2$.

(2) Every closed ideal having finite codimension in B has a bounded left (or right) approximate identity.

Then any module derivation from $B$ into a Banach-B-bimodule is continuous.

Proof. Let $D$ be a module derivation from $B$ into a Banach- $B$ bimodule $M$, and let $I_{L}$ be the left continuity ideal for $D$. Suppose $I_{L}$ is of infinite codimension in $B$. We obtain a contradiction by applying the remark after Lemma 1 with $T_{n} x=b_{n} x$ for all $x$ in $B, R_{n} y=b_{n} \cdot y$ and $U_{n} y=c_{n} \cdot y$ for all $y$ in $M$, and noting the formula $\left(D T_{n}-R_{n} D\right)(x)=$ $D\left(b_{n} x\right)-b_{n} \cdot D(x)=D\left(b_{n}\right) \cdot x$. Hence $I_{L}$ is a closed ideal having finite codimension in $B$, and so has a bounded left (or right) approximate identity. Thus $D$ is continuous on $B$.

REMARKs. (a) $C^{*}$-algebras and the group algebra of a locally compact abelian group satisfy the hypotheses of Theorem 2 .

(b) We can replace condition (1) by the stronger one that every closed ideal $K$ of infinite codimension in $B$ has the property that given $b$ in $B \backslash K$, there exists $a, c$ in $B$ such that $a b \notin K, b c \notin K$ but $a b c \in K$. A simple inductive argument shows that this implies the condition in the theorem. If $B$ is commutative this is merely saying that for each $b$ in $B \backslash K$, the annihilator of $b+K$ in the quotient algebra $B / K$ is not prime. However $C^{*}$-algebras no longer have this property in general e.g. take $B=C([0,1] \cup\{2\})$ and $K$ the zero ideal. A. M. Davie has pointed out that for a Hilbert space $H, K=K(H)$, the ideal of compact operators on $H$, does have this property in $B(H)$, and it is clear that if $B$ is a $C^{*}$-algebra and $K$ is an ideal in $B$ such that $B / K$ has no nontrivial idempotents then $K$ has this property.

Bade and Curtis [2] have also obtained sufficient conditions on a Banach algebra $B$ so that every module derivation from $B$ is continuous. One of their conditions is identical to (2) in Theorem 
2. The other is as follows: if $K$ is a closed ideal of infinite codimension in $B$, then there exists a sequence $\left\{x_{n}\right\}$ in $B$ satisfying $x_{n} x_{m}=0(n \neq m)$ and $x_{n}^{2} \notin K$ for all $n$. We remark here that the two theorems are in fact different and Theorem 2 appears to cover a wider class of algebras. To show this we use the example $L^{1}[0,1]$ which satisfies the conditions of Theorem 2 (which implies that every module derivation from $L^{1}[0,1]$ is continuous - we obtain this result most easily as a corollary to Theorem 5 as will be shown). For the closed ideals of $L^{1}[0,1]$ are of the form $M(\alpha)=\left\{f \in L^{1}[0,1]: f=0\right.$ a.e. on $\left.[0, \alpha]\right\}$ for some $\alpha$ with $0 \leqq \alpha \leqq 1$ [6]. Let $f(\beta)$ be the characteristic function of $[\beta, 1]$ for each $\beta$ in $[0,1]$. If $\alpha$ and $\beta$ are positive and $\alpha+\beta \leqq 1$, then $f(\alpha) f(\beta) \in M(\alpha+\beta)$. Let $M(\alpha)$ be a closed ideal of infinite codimension in $L^{1}[0,1]$. Then $\alpha>0$. Let $g \in L^{1}[0,1], g \notin M(\alpha)$. Let $p=$ $\inf \{q: g \in M(q)\}$. Then $0 \leqq p<\alpha$. We choose positive real numbers $\beta, \quad \gamma$ so that $p+\beta<\alpha, \quad p+\gamma<\alpha$ but $\alpha<p+\beta+\gamma \leqq 1$. Then $f(\beta) g \notin M(\alpha), f(\gamma) g \notin M(\alpha)$ but $f(\beta) g f(\gamma) \in M(\alpha)$. Remark (b) after Theorem 2 shows that condition (1) is satisfied. The only closed ideal of $L^{1}[0,1]$ having finite codimension in $L^{1}[0,1]$ is $L^{1}[0,1]$ itself which has a bounded approximate identity (take a one-sided Dirac sequence) and so condition (2) is also satisfied. However $L^{1}[0,1]$ does not satisfy the condition on closed ideals of infinite codimension given by Bade and Curtis. For let $M(\alpha)$ be a closed ideal of $L^{1}[0,1]$ where $0<\alpha<$ $\frac{1}{2}$. Then $M(\alpha)$ is of infinite codimension. Suppose there exists a sequence $\left\{x_{n}\right\}$ in $L^{1}[0,1]$ with $x_{n} x_{m}=0(n \neq m)$ and $x_{n}^{2} \notin M(\alpha)$ for all $n \geqq 1$. Let $\beta_{n}=\inf \left\{\beta: x_{n} \in M(\beta)\right\}(n \geqq 1)$. It is clear that $0<\beta_{n} \leqq \alpha$ and $\beta_{n}+\beta_{m} \geqq 1(n \neq m)$. Let $\gamma=\liminf _{n \rightarrow \infty}\left\{\beta_{n}\right\}$. Then $\beta_{j} \geqq 1-\gamma$ for all $j \geqq 1$ which shows that $\gamma \geqq 1-\gamma$ i.e. $\gamma \geqq \frac{1}{2}$. But $0<\beta_{n} \leqq \alpha<\frac{1}{2} \Rightarrow$ $\gamma<\frac{1}{2}$ which yields the required contradiction.

The next lemma, which follows from Lemma 1, is due to W. G. Bade and P. C. Curtis, Jr. [3], and is closely related to Theorem 3.3 of [16].

LEMMA 3. Let $B$ be a commutative Banach algebra with identity and $M$ a Banach-B-bimodule. Let $D: B \rightarrow M$ be a discontinuous module derivation. Then there exists $x_{0}$ in $B$ such that if $D_{0}: B \rightarrow M$ is given by $D_{0}(b)=x_{0} \cdot D(b)$ for all $b$ in $B$, we have that $I_{0}$, the continuity ideal of $D_{0}$, is a closed prime ideal of $B$.

REMARK. We can assume that $B$ does not have an identity by forming the algebra $B \oplus \lambda C$, extending $D$ by $D(\lambda 1)=0$, and allowing $x_{0}$ to be in $B \oplus \lambda C$. $\quad I_{0}$ would then be a prime ideal in $B \oplus \lambda C$ with $I_{0}^{\prime}=\left\{b \in B:(b, 0) \in I_{0}\right\}$ a prime ideal in $B$.

For the next theorem we will require the following particular case of a theorem of Loy [13] (see also [5]). 
Proposition 4. Let $A$ be a separable Banach algebra such that $A^{2}$ is closed in $A$. Then there is a constant $K$ and an integer $m$ such that if $a \in A^{2}$ there exist $a_{j}, b_{j} \in A, 1 \leqq j \leqq m$, satisfying
(i) $a=\sum_{j=1}^{m} a_{j} b_{l}$
(ii) $\sum_{j=1}^{m}\left\|a_{j}\right\|\left\|b_{j}\right\| \leqq K\|a\|$.

In the separable, commutative case we can now prove the following theorem.

THEOREM 5. Let $B$ be a commutative separable Banach algebra such that $B^{2}$ is of finite codimension in $B$ and which satisfies the following two conditions:

(1) There are no closed prime ideals of infinite codimension.

(2) Every maximal ideal $M$ of $B$ has $M^{2}$ of finite codimension in $B$. Then any module derivation from $B$ into a Banach-B-bimodule is continuous.

Proof. Without loss of generality assume that $B$ has an identity. Suppose that $D$ is a discontinuous module derivation from $B$ into some Banach- $B$-bimodule $M$. Let $D_{0}, I_{0}$ be as given in Lemma 3. $D_{0}$ is then also discontinuous. $I_{0}$ is a closed prime ideal and so must be of finite codimension. But a prime ideal of finite codimension is maximal and so $I_{0}^{2}$ is of finite codimension in $B$.

But then $I_{0}^{2}$ must be closed since for a separable Banach algebra $A$, $A^{k}$ of finite codimension for some integer $k \geqq 2 \Rightarrow A^{k}$ is closed $[5,13]$. We now obtain a contradiction by showing that $D_{0}$ is continuous on $I_{0}^{2}$. Let $f \in I_{0}^{2}$. Proposition 4 gives $m \in N$ and $K>0$ such that $f=\sum_{j=1}^{m} g_{j} h_{,} \quad$ (where $g_{j}, \quad h_{j} \in I_{0}, \quad 1 \leqq j \leqq m$ ) and $\sum_{j=1}^{m}\left\|g_{j}\right\|\left\|h_{j}\right\| \leqq$ $K\|f\|$. Then

$$
\begin{aligned}
\left\|D_{0}(f)\right\|=\left\|\sum_{j=1}^{m} D_{0}\left(g_{j} h_{j}\right)\right\| & \leqq \sum_{j=1}^{m}\left\|D_{0}\left(g_{j}\right) \cdot h_{j}+g_{j} \cdot D_{0}\left(h_{j}\right)\right\| \\
& \leqq \sum_{j=1}^{m} 2 M\left\|g_{\jmath}\right\|\left\|h_{j}\right\|,
\end{aligned}
$$

where $M$ is a constant (see Lemma 1.3 (iii) of [15]), and then $\left\|D_{0}(f)\right\| \leqq$ $2 M \sum_{j=1}^{m}\left\|g_{\text {, }}\right\|\left\|h_{j}\right\| \leqq 2 M K\|f\|$ which concludes the proof.

Remarks. (1) Given a particular module $M$ we can weaken condition (1) slightly to "there are no closed prime ideals of infinite codimension in $B$ which annihilate some nontrivial submodule of $M$ ".

(2) Condition (2) is best possible since if $B$ has a maximal ideal $M$ such that $M^{2}$ is not of finite codimension in $B$ then there is a discontinuous module derivation from $A$ into $C[15, p .49]$. Condition (2) does not 
suffice by itself, for there is a discontinuous module derivation from the disc algebra (see [15, Theorem 8.9]).

The following corollary extends Corollary 4 of [8].

COROllary 6. Module derivations from $L^{1}[0,1]$ into a Banach$L^{1}[0,1]$-module are continuous.

Proof. $L^{1}[0,1]$ is commutative and separable and has no closed prime ideals and no maximal ideals. The result follows from Theorem 5.

REMARK. The methods of this section can also be used to obtain results on module homomorphisms.

3. Higher derivations. R. J. Loy has shown that the result of B. E. Johnson and A. M. Sinclair [9] giving the automatic continuity of derivations on semi-simple Banach algebras can be extended to higher derivations whose domain algebra is the same as the range algebra and where $F_{0}$ is the identity map. He did this by using results of Heerema [7] to express the higher derivations in terms of derivations. We extend this result (i) by allowing the domain algebra to be any Banach algebra whatsoever, (ii) by allowing the range algebra to include a wider class than just semi-simple algebras and (iii) by weakening the condition that $F_{0}$ be the identity map.

THEOREM 7. Let $B$ be a Banach algebra with the property that for each infinite-dimensional closed ideal $J$ in $B$ there is a sequence $\left\{b_{n}\right\}$ in $B$ such that $\left(J b_{n} \cdots b_{1}\right)^{-} \supset\left(J b_{n+1} \cdots b_{1}\right)^{-}$for all positive integers $n$. Suppose also that $B$ contains no nonzero finite-dimensional nilpotent ideals. Let $\left\{F_{n}\right\}$ be a higher derivation of any rank from a Banach algebra $A$ onto $B$ such that $\operatorname{Ker} F_{0} \subseteq \operatorname{Ker} F_{n}$ for all $n$. Then $\left\{F_{n}\right\}$ is continuous.

Proof. We prove that $F_{n}$ is continuous for all $n$ by induction. Clearly $F_{0}$ is a homomorphism and since $F_{0}$ is onto $B$ it is continuous by Theorem 2 of [8]. Now assume that $F_{n}$ is continuous for $0 \leqq n \leqq k-1$. We have

$$
F_{k}(x y)=\sum_{i=0}^{k} F_{i}(x) F_{k-i}(y) \quad \text { for } x, y \in A .
$$

Hence $F_{k}(x y)-F_{0}(x) F_{k}(y)=\sum_{i=1}^{k} F_{i}(x) F_{k-1}(y)$. For a fixed $x$, we then have $\left(F_{k} L(x)-L\left(F_{0}(x)\right) F_{k}\right)(y)=K(y)$ where $K$ is continuous by the inductive hypothesis, and $L(a)$ denotes the operation of left multiplication. 
So, for a fixed $x, F_{k} L(x)-L\left(F_{0}(x)\right) F_{k}$ is continuous $\cdots(1)$

Since $F_{0}$ is onto and given the inductive hypothesis we have that $\Theta\left(F_{k}\right)$ is a closed ideal in $B$. Then, using (1), in an identical fashion to the proof of Theorem 2 in [8] it follows that $\subseteq\left(F_{k}\right)$ is finitedimensional. The radical of $\subseteq\left(F_{k}\right)$ is a finite-dimensional nilpotent ideal in $B$, and so is zero. Then since $\widetilde{S}\left(F_{k}\right)$ is a finite-dimensional semisimple algebra it has an identity $e$ [4, p. 135]. The identity of an ideal in an algebra is a central idempotent in the algebra. Choose $h \in$ $F_{0}^{-1}\{e\} . \quad F_{0}\left(h^{2}-h\right)=0$ and so $F_{f}(h)=F_{j}\left(h^{2}\right)(j=1, \cdots, k)$. This gives $F_{j}(h)=0 \quad(j=1, \cdots, k)$. Let $M=\operatorname{Ker} F_{0}$ and consider the algebra $h A / M$. Define $F_{0}^{1}: h A / M \rightarrow e B$ by $F_{0}^{1}(h a+M)=e F_{0}(a) . \quad F_{0}^{1}$ is oneone and onto $e B$ which is finite-dimensional and so $h A / M$ is finite-dimensional. Define $F_{k}^{1}: h A / M \rightarrow e B$ by $F_{k}^{1}(h a+M)=e F_{k}(a)$ which is well-defined since $F_{J}(h)=0(j=1, \cdots, k) . \quad F_{k}^{1}$ is continuous since $h A / M$ is finite-dimensional. Now take $y \in \mathbb{S}\left(F_{k}\right)$. There exist $x_{n} \rightarrow 0$ in $A$ with $F_{k}\left(x_{n}\right) \rightarrow y$. Then $F_{k}^{1}\left(h x_{n}+M\right)=e F_{k}\left(x_{n}\right) \rightarrow e y=$

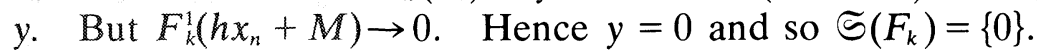

COROLlary 8. (i) Higher derivations $\left\{F_{n}\right\}$ of any rank from any Banach algebra onto $L^{1}[0,1]$ are continuous if $\operatorname{Ker} F_{0} \subseteq \operatorname{Ker} F_{n}$ for all $n$.

(ii) Higher derivations $\left\{F_{n}\right\}$ of any rank from any Banach algebra onto semi-simple Banach algebras are continuous if $\operatorname{Ker} F_{0} \subseteq \operatorname{Ker} F_{n}$ for all $n$.

Proof. Semi-simple Banach algebras and $L^{1}[0,1]$ satisfy the hypotheses of Theorem 7 [8].

Remarks. (a) The remarks after Theorem 2 of [8] apply in this case also.

(b) The result for Banach algebras (such as $L^{1}[0,1]$, Banach algebras of power series, weighted convolution algebras) which satisfy the hypothesis on infinite-dimensional closed ideals and for which there are no nonzero finite-dimensional ideals can be proved in the same fashion without requiring the assumption on the kernels.

(c) For Banach algebras of power series the continuity of higher derivations under the restricted conditions of $A=B, F_{0}$ the identity map was first proved by R. J. Loy [11].

(d) Using the methods of [7] and [10] it is possible to classify all the higher derivations acting on $L^{1}[0,1]$ where $F_{0}$ is the identity map.

(e) The methods of the proof also give the continuity of higher derivations on $n$ indices of $A$ into $B$ (see [11]) under similar hypotheses to Theorem 7 .

EXAMPLE. The following example from Loy [12] shows that we 
require some assumption of the type given on $\operatorname{Ker} F_{0}$. Take $A=l^{2}$ with identity adjoined and pointwise product and $B=C$. Let $\phi$ be a character on $A$ with kernel $l^{2}$ and let $\theta$ be a discontinuous linear functional on $l^{2}$ which vanishes on the dense subset $l^{1}=\left(l^{2}\right)^{2}$. Extend $\theta$ to $A$ by $\theta(1)=0$ and linearity. Then $F_{0}=\phi, F_{i}=0,1 \leqq i \leqq n-1$, $F_{n}=\theta$ is a higher derivation of rank $n$ of $A$ onto $B$ with $F_{n}$ discontinuous. Here $\operatorname{Ker} F_{0} \not \subset \operatorname{Ker} F_{n}$.

\section{REFERENCES}

1. G. R. Allan, Embedding the algebra of formal power series in a Banach algebra, Proc. London Math. Soc., 25 (1972), 329-340.

2. W. G. Bade and P. C. Curtis, Jr., The continuity of derivations of Banach algebras, J. Functional Analysis, 16 (1974), 372-387.

3. W. G. Bade and P. C. Curtis, Jr., (to appear in J. Functional Analysis).

4. F. F. Bonsall and J. Duncan, Complete Normed Algebras, Springer-Verlag, Berlin, 1973.

5. J. P. R. Christensen, Codimension of some subspaces in a Frechet algebra, Proc. Amer. Math. Soc., 57 (1976), No. 2, 276-278.

6. W. F. Donoghue, The lattice of invariant subspaces of a completely continuous quasinilpotent transformation, Pacific J. Math., 7 (1957), 1031-1035.

7. N. Heerema, Derivations and embeddings of a field in its power series ring, Proc. Amer. Math. Soc., 11 (1960), 188-194.

8. N. P. Jewell and A. M. Sinclair, Epimorphisms and derivations on $L^{1}[0,1]$ are continuous, Bull. London Math. Soc., 8 (1976), 135-139.

9. B. E. Johnson and A. M. Sinclair, Continuity of derivations and a problem of Kaplansky, Amer. J. Math., 90 (1968), 1067-1073.

10. H. Kamowitz and S. Scheinberg, Derivations and automorphisms of $L^{1}[0,1]$, Trans. Amer. Math. Soc., 135 (1969), 415-427.

11. R. J. Loy, A class of topological algebras of formal power series, Carleton Mathematical Series No. 11, November 1969.

12. _ Continuity of higher derivations, Proc. Amer. Math. Soc., 37 (1973), 505-510.

13. - Multilinear mappings and Banach algebras, preprint).

14. J. R. Ringrose, Automatic continuity of derivations of operator algebras, J. London Math. Soc., (2), 5 (1972), 432-438.

15. A. M. Sinclair, Automatic continuity of linear operators, London Math. Soc., Lecture Note Series, 21 (1976).

16. Homomorphisms from $C_{0}(R)$, J. London Math. Soc., (2), 11 (1975), 165-174.

Received March 25, 1976.

UNIVERSITY OF Edinburgh, SCOTLAND.

Present address: UNIVERSITY OF CALIFORNIA

Berkeley, California 94720 



\section{Pacific Journal of Mathematics}

Vol. 68, No. 1

March, 1977

Richard Julian Bagby, On $L^{p}, L^{q}$ multipliers of Fourier transforms . .......

Robert Beauwens and Jean-Jacques Van Binnebeek, Convergence theorems in

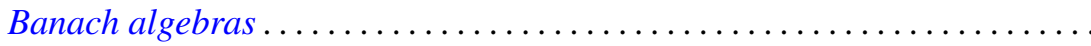

James Cyril Becker, Skew linear vector fields on spheres in the stable

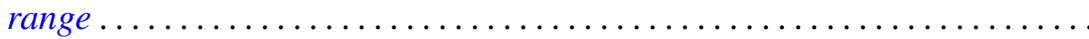

Michael James Beeson, Continuity and comprehension in intuitionistic formal

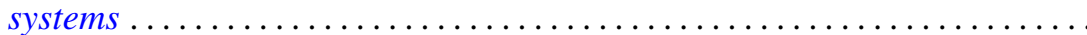

James K. Deveney, Generalized primitive elements for transcendental field

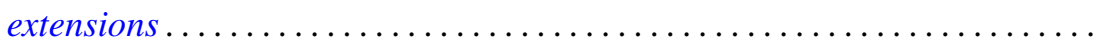

Samuel S. Feder, Samuel Carlos Gitler and K. Y. Lam, Composition properties

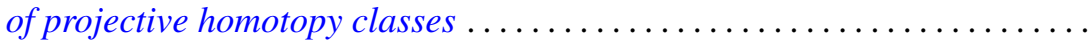

Nathan Jacob Fine, Tensor products of function rings under composition ......

Benno Fuchssteiner, Iterations and fixpoints . . . . . . . . . . . . . .

Wolfgang H. Heil, On punctured balls in manifolds

Shigeru Itoh, A random fixed point theorem for a multivalued contraction

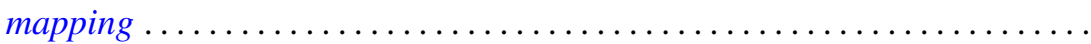

Nicolas P. Jewell, Continuity of module and higher derivations . . . . . . ......

Roger Dale Konyndyk, Residually central wreath products . . . . . . . . . . .

Linda M. Lesniak and John A. Roberts, On Ramsey theory and graphical

parameters.

Vo Thanh Liem, Some cellular subsets of the spheres.

Dieter Lutz, A perturbation theorem for spectral operators

P. H. Maserick, Moments of measures on convex bodies ... . . .

Stephen Joseph McAdam, Unmixed 2-dimensional local domains . .

D. B. McAlister and Norman R. Reilly, E-unitary covers for inverse semigroups...

William H. Meeks, III and Julie Patrusky, Representing codimension-one

homology classes by embedded submanifolds . . .

Premalata Mohapatro, Generalised quasi-Nörlund summability . .

Takahiko Nakazi, Superalgebras of weak-*Dirichlet algebras .

Catherine Louise Olsen, Norms of compact perturbations of operators .

William Henry Ruckle, Absolutely divergent series and isomorphism of

subspaces. II.

Bernard Russo, On the Hausdorff-Young theorem for integral operators .

Arthur Argyle Sagle and J. R. Schumi, Anti-commutative algebras and

homogeneous spaces with multiplications ............

Robert Evert Stong, Stiefel-Whitney classes of manifolds .

D. Suryanarayana, On a theorem of Apostol concerning Möbius functions of

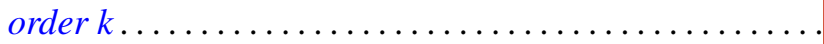

Yoshio Tanaka, On closedness of $C$ - and $C^{*}$-embeddings . . 